\title{
Phytothérapie traditionnelle par Acalypha indica induisant un accident hémolytique chez les patients porteurs d'un déficit en G6PD : une circonstance fréquente à Mayotte ?
}

\author{
Plant Traditional Treatment with Acalypha indica Inducing Haemolysis in Patients with G6PD \\ Deficiency: A Frequent Circumstance in Mayotte?
}

\author{
P. Durasnel - C. Tantet $\cdot$ A. Chamouine $\cdot$ R Blondé \\ Reçu le 29 décembre 2017; accepté le 14 mars 2018 \\ (C) Société de pathologie exotique et Lavoisier SAS 2018
}

\begin{abstract}
Résumé Une phytothérapie traditionnelle par des remèdes contenant Acalypha indica L. est susceptible d'induire un accident hémolytique intravasculaire, potentiellement grave, chez les patients déficitaires en glucose-6-phosphatedéshydrogénase (G6PD). La toxicité potentielle de cette plante est connue, mais peu diffusée. Dans les régions où elle pousse et où coexistent un recours fréquent à la médecine traditionnelle et une prévalence élevée du déficit en G6PD, elle devrait être systématiquement recherchée. La situation semble fréquente à Mayotte, et n'avait jusqu'alors été signalée que rarement au Sri Lanka. Il semble indispensable que dans ces régions, les patients ou leurs parents soient systématiquement informés du risque potentiel en cas de recours à une médecine traditionnelle par les plantes, en plus des facteurs déclenchants habituellement recherchés.
\end{abstract}

Mots clés Hémolyse · Déficit en G6PD · Acalypha indica · Médecine traditionnelle $\cdot$ Plante $\cdot$ Mayotte $\cdot$ Comores .

Océan Indien

\begin{abstract}
A traditional treatment by plants with Acalypha indica $\mathrm{L}$. can induce an intravascular haemolysis in patients with a glucose-6-phosphate-dehydrogenase (G6PD) deficiency. This information is poorly diffused in areas where the plant grows, where it is consumed for ethnomedicinal
\end{abstract}

P. Durasnel $(\bowtie) \cdot R$ Blondé

Service de réanimation polyvalente,

Centre hospitalier de Mayotte

e-mail : p.durasnel@chmayotte.fr

C. Tantet

Service de médecine interne,

Centre hospitalier de Mayotte

A. Chamouine

Service de pédiatrie, Centre hospitalier de Mayotte purpose and where G6PD deficiency prevalence is high; as a consequence, the probability of haemolytic accidents is presumably underestimated. It seems frequent in Mayotte according to local recent data reporting. Such accidents were previously only, and on a rare basis, reported in Sri Lanka. It seems necessary, at least in Mayotte, to inform patients, or the patients' relatives, about the potential risk in case of using traditional medicine by plants, in addition to all other circumstances able to induce haemolysis in G6PD deficiency.

Keywords Haemolysis - G6PD deficiency · Acalypha indica $\cdot$ Traditional medicine $\cdot$ Plant $\cdot$ Mayotte $\cdot$ Comoros $\cdot$ Indian Ocean

\section{Introduction}

Dans les pays développés, les intoxications par les plantes sont le plus souvent accidentelles, et concernent essentiellement les enfants, pour de faibles quantités ingérées, avec une morbidité et une mortalité faible. Les intoxications les plus graves surviennent le plus souvent dans un contexte suicidaire ou addictif. Dans les pays en développement, où les populations ont recours aux médecines traditionnelles à la place ou simultanément à la médecine conventionnelle, les intoxications par les plantes sont plus fréquentes et cause d'une morbidité et d'une mortalité mal connues, mais probablement supérieure et sous-estimée. À Mayotte, des accidents liés à des traitements traditionnels par les plantes ont été récemment décrits [2,3]. À la suite de ces observations, une sensibilisation des équipes médicales du Centre hospitalier de Mayotte, et une collecte prospective des cas suspects, ont permis d'identifier plusieurs accidents liés à l'utilisation d'Acalypha indica L (Fig. 1). Si la toxicité potentielle de cette plante est connue, mais peu diffusée, son usage 
traditionnel à Mayotte n'était jusqu'alors pas connu des praticiens, ni sa responsabilité identifiée dans des accidents hémolytiques.

\section{Contexte clinique}

L'utilisation de l'Acalypha indica en médecine traditionnelle a été reconnue récemmnent (début 2006) à Mayotte chez une patiente adulte en réanimation, hospitalisée pour une anémie hémolytique aiguë grave. Un déficit en glucose6-phosphate-déshydrogénase (G6PD) a été confirmé ultérieurement chez cette patiente. Depuis ce cas index, la recherche de la prise récente de cette plante en cas d'hémolyse, est faite systématiquement au Centre hospitalier de Mayotte. Sur une période récente de 24 mois, 5 autres cas certains ont été attestés : trois chez des enfants et deux chez des adultes...

Une étude prospective en cours au service de pédiatrie ( « mayG6PD») a permis d'identifier 24 enfants admis pour hémolyse aiguë et porteurs d'un déficit confirmé en G6PD, du 1/1/2012 au 31/12/2017 (72 mois). Dans dix cas au moins, un traitement traditionnel par les plantes avait été pris dans les jours précédents. La prise d'Acalypha indica L n'a pu être confirmée que dans trois cas parmi les plus récents, mais il n'a pas été possible à ce jour d'investiguer les cas plus anciens.
Depuis début 2016, un autre adulte, après le cas index a été hospitalisé en réanimation et un autre cas en service de médecine pour hémolyse aiguë, avec déficit en G6PD confirmé a posteriori. Dans ces deux cas, la prise d'Acalypha indica $\mathrm{L}$ dans les jours précédents est certaine.

Chez les 3 adultes et chez 2 enfants, le déficit en G6PD n'était pas connu avant la crise hémolytique concernée.

Dans tous les cas où la consommation d'Acalypha indica L est attestée, l'interrogatoire ne retrouvait pas d'autre élément déclenchant potentiel. Cette consommation se plaçait toujours dans un contexte d'affection respiratoire (asthme, bronchite, toux, etc.), qui semble donc l'indication la plus fréquemment retenue par les tradipraticiens mahorais.

\section{Discussion}

Le déclenchement de crises hémolytiques, par l'absorption de préparations à base d'Acalypha indica L., a été décrit initialement par des auteurs sri lankais [4] en 1994. Cette plante, très commune à Mayotte, est appelée en français " ricinelle des Indes », " oreille de chatte ", ou " herbe chatte », en anglais « indian nettle » ou « three-seeded mercury ». Elle porte à Mayotte les noms vernaculaires de « pvahara pefu » en shimaoré (dialecte swahili) ou « haiti ambadriha » en kibushi (dialecte malgache). C'est une plante

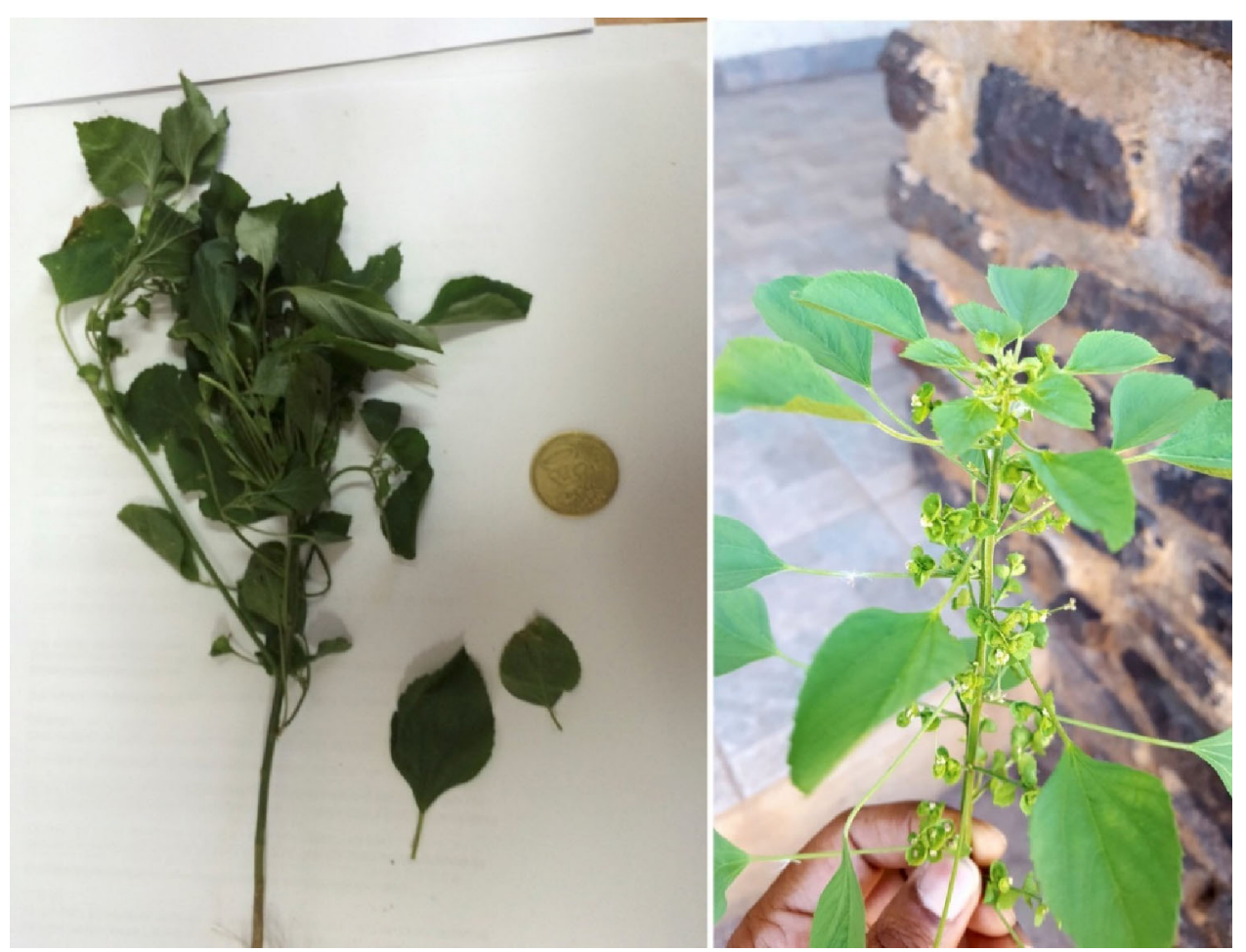

Fig 1 Acalypha indica L., à gauche plante ramenée par la famille d'un patient (photo Ph. DURASNEL), à droite plante en fleur sur pied (photo A. Chamouine) / Acalypha indica L., Left: plant collected and brought back by a patient's relatives (photography Ph. DURASNEL). Right: growing plant with flowers (photography A. Chamouine) 
dicotylédone de la famille des Euphorbiacées. Elle est rudérale, présente dans les régions tropicales, subtropicales et tempérées chaudes. On la rencontre en Afrique centrale et australe. Elle est également répandue dans les îles de l'océan Indien, en Inde, en Asie du Sud-Est et en Océanie. Elle a été introduite dans les régions chaudes du Nouveau Monde, mais ne pousse pas en Europe.

Son usage est très commun en médecine ayurvédique, mais semble l'être également dans d'autres régions, comme les Comores. Elle est, dans de nombreux autres pays où on la rencontre, utilisée en médecine traditionnelle, pour des indications diverses : en collyre pour les conjonctivites (Afrique de l'Est), en cataplasme sur des plaies infectées (Namibie), en décoction et massage pour les douleurs articulaires (Comores), en infusion pour l'asthme (Mayotte, la Réunion, Seychelles), comme purgatif et vermifuge (Madagascar), en usage local pour traiter la gale et d'autres problèmes cutanés (Maurice). Elle faisait jadis partie de la pharmacopée britannique. Elle a de nombreux usages médicinaux dans le sous-continent indien, et figure dans la pharmacopée indienne comme expectorant pour traiter l'asthme et la pneumonie. Elle contient un hétéroside cyanogénétique, l'acalyphine, mais aussi des flavonoïdes, des tanins, et des alcaloïdes.

La consommation fréquente d'Acalypha indica $\mathrm{L}$. et la prévalence élevée du déficit en G6PD dans la population générale de Mayotte indiquent que la fréquence des accidents hémolytiques induits par ce remède traditionnel est probablement sous-estimée. Les crises hémolytiques observées étaient d'intensité variable, mais ont conduit quatre fois le patient en réanimation. L'évolution a été favorable dans tous les cas, mais parfois longue et difficile. La recherche du déficit en G6PD était positive chez tous les patients,

Le génotype variant G6PD A-, le plus commun en Afrique, est retrouvé chez tous les patients ayant pu être génotypés. Plus précisément à Mayotte il s'agit du variant G6PD A- 202A. Le variant G6PD A- est responsable dans la majorité des cas d'accident lors d'un stress oxydatif, avec un déficit modéré (10-60\%, classe III OMS).

Acalypha figure sur le document de la Direction générale de la santé [1] d'information et de conseils en cas de déficit en G6PD datant de 2008. Aucune mention concernant les plantes médicinales ne figure sur le protocole national de diagnostic et de soins (PNDS) concernant le déficit en G6PD édité en 2017 et diffusé par la Haute autorité de santé (HAS).

\section{Conclusion}

Acalypha indica L., utilisé dans le cadre d'une médecine traditionnelle par les plantes, est susceptible d'induire un accident hémolytique grave chez des sujets déficitaires en G6PD. Sur les données rapportées ici, cette situation semble fréquente à Mayotte où coexistent un fréquent usage de la plante en médecine traditionnelle et une prévalence élevée de déficit en G6PD. Ces circonstances imposent à Mayotte une information ciblée des patients connus déficitaires en G6PD ou de leur entourage. Il est possible que d'autres territoires soient concernés, dont les départements français d'outre-mer. Les effets secondaires de cette plante sont connus mais très peu diffusés, et méritent d'être recherchés, la situation pouvant être rencontrée ailleurs qu'à Mayotte, y compris peut-être en métropole avec la pratique croissante des médecines dites « naturelles » avec des plantes importées.

Remerciement Remerciements à Luc Vanhuffel, botaniste, pour son aide toujours aussi précieuse dans l'identification des plantes médicinales proposée par le service de réanimation du Centre hospitalier de Mayotte

Liens d'intérêt : Les auteurs déclarent ne pas avoir de liens d'intérêts.

\section{Bibliographie}

1. Direction générale de la santé, Hôpital Necker enfants malades (2008) Information et conseils. Déficit en glucose-6-phosphate déshydrogénase (G6PD) ou favisme, $8 \mathrm{p}$

2. Durasnel P, Vanhuffel L, Blondé R, et al (2014) Intoxications graves lors de traitements traditionnels par les plantes à Mayotte. Bull Soc Pathol Exot 107:306-11. doi: 10.1007/s13149-014-0400-7 [http://www.pathexo.fr/documents/articles-bull/131490306.pdf]

3. Perinet I, Loison E, Tichadou L, et al (2011) Ingestion volontaire de décoction de henné (Lawsonia inermis) à l'origine d'une anémie hémolytique chez une patiente atteinte d'un déficit en G6PD. Méd Trop 71:292-4

4. Lamabadusuriya SP, Jayantha UK (1994) Acalypha indica induced haemolysis in G6PD deficiency. Ceylon Med J 39:46-7 